\title{
Direct determination of free amino acids and sugars in green tea by anion-exchange chromatography with integrated pulsed amperometric detection
}

\author{
Yongsheng Ding, Hong Yu, Shifen Mou* \\ Research Center for Eco-Environmental Sciences, Chinese Academy of Sciences, P.O. Box 2871, Beijing 100085, China
}

Received 5 July 2002; received in revised form 1 October 2002; accepted 2 October 2002

\begin{abstract}
Determination of amino acids and sugars in green tea by anion-exchange chromatography with integrated pulsed amperometric detection was developed. Amino acids and sugars were separated on an anion-exchange column at a flow-rate of $0.25 \mathrm{ml} / \mathrm{min}$ by using ternary gradient elution consisting of deionized water, $0.25 \mathrm{M}$ sodium hydroxide, and $1.0 \mathrm{M}$ sodium acetate. Under optimized conditions, theanine was separated from glutamine and three sugars (glucose, fructose, and sucrose) were eluted earlier than the neutral amino acids to avoid their interference with each other. RSDs of the peak area of analytes were lower than $4.6 \%$. Detection limits for the analytes ranged from 0.12 to $4.9 \mathrm{pmol}$. The linearities for all analytes were two or three orders of magnitude with the correlation coefficients greater than 0.99. This method was applied to determination of amino acids and sugars in green tea with satisfactory results.
\end{abstract}

(C) 2002 Elsevier Science B.V. All rights reserved.

Keywords: Tea; Food analysis; Amino acids; Carbohydrates

\section{Introduction}

Tea (tea infusion) is now one of the most popular drinks in the world, and its consumption nearly ranks with that of coffee. According to the degree of fermentation, tea is classified into green tea (unfermented), oo-long tea (semi-fermented), and black tea (fully fermented). Tea infusion not only gives specific taste and flavor, it also has many physiological and functional effects due to the fact that it contains such compounds as polyphenols, caffeine,

\footnotetext{
*Corresponding author. Tel.: +86-10-6284-9182; fax: +86-106284-9239.

E-mail address: shifenm@mail.rcees.ac.cn (S. Mou).
}

amino acids, vitamins, carbohydrates, etc. [1-3]. The total content of amino acids in green tea is the greatest in all kinds of tea. Many studies have demonstrated the relationship between the green tea quality and the content of amino acids in green tea $[4,5]$. Actually, there is high correlation between the green tea quality and content of amino acids, especially that of theanine. Theanine (5- $N$-ethylglutamine) exists only in the free (non-protein) form and is the most important free amino acid in tea [6]. Besides having a delicate taste, theanine also has many biological effects. For example, it has been reported that theanine can decrease the level of norepinephrine and serotonin in brain, and intake of theanine by hypertensive rats results in decreased 
blood pressure [7]. Recently, cooperative effects of anti-tumor agents and theanine on cancer have also been reported [8].

There are many descriptions of procedures for analysis of amino acids in green tea based on liquid chromatography with post-column or pre-column derivatization [6,9-11]. Relative complexity of the reaction system, high cost of maintenance of postcolumn derivatization, low stability of amino acid derivatives, or reagent interference in pre-column derivatization are among the shortcomings of these methods. Additionally, analysis of theanine together with other compounds was reported by means of capillary electrophoresis (CE) and micellar electrokinetic capillary chromatography (MECC) using UV absorbance detection [12,13]. Since theanine is lacking a suitable chromophore and UV detection has to be carried out at short wavelengths (190-200 nm), the reported CE and MECC techniques are plagued by interference and poor sensitivity.

Recently, there have been several reports describing the use of integrated amperometric detection (IPAD) without derivatization for amino acids and carbohydrates in conjunction with high-pH anionexchange chromatographic (AEC) separation. Clarke et al. [14] described an optimized waveform (waveform: changes of potential applied to a gold working electrode vs. time) capable of detecting all primary and secondary amino acids. The optimized waveform keeps the gold working electrode active without causing any electrode corrosion or loss of signal. Generally speaking, amino acids accompanied by carbohydrates exist in many actual samples. The interference between carbohydrates and amino acids can be avoided only if the amounts of amino acids are approximately equal to those of sugars. Samples containing a larger amount of carbohydrates and trace of amino acids (molar ratio $>$ ca. 50 to 1 ) are obviously challenging for the AEC-IPAD method. Jandik et al. [15] introduced a technique to eliminate carbohydrates from the samples by using a cationexchanger in hydrogen form for sample preparation. The same group of authors also developed a bimodal waveform for identifying amino acids and carbohydrates in co-eluting peaks [16]. In our opinion, the above two methods do not represent a suitable solution for all types of samples, lower recoveries of certain amino acids and the inability to quantify amino acid and carbohydrate in co-eluting peaks still represents a problem for some of the samples.

The objective of our work was to devise an AECIPAD method for determination of amino acids and sugars in green tea. Our aim was to separate these compounds fully and avoid any interference with each other. We developed new gradient conditions eluting amino acids and sugars at different retention time regions. The new gradient conditions were successfully applied to the analysis of amino acids and sugars in the infusions of green tea.

\section{Experimental}

\subsection{Chemicals}

Theanine standard was purchased from Tokyo Kasei (Tokyo, Japan). Other amino acid standards were purchased from KangDa Chemicals (Shanghai, China). Three sugars (glucose, fructose, and sucrose) were purchased from Sigma-Aldrich (St. Louis, MO, USA). Sodium hydroxide (low carbonate) was purchased from Beijing Chemical (Beijing, China). Anhydrous sodium acetate was obtained from Dionex (Sunnyvale, CA, USA). Standard mixtures were made by diluting each of the stock solutions to desired concentrations with a diluent containing $20 \mathrm{mg} / 1$ sodium azide. The $50 \%$ sodium hydroxide was prepared by dissolving solid sodium hydroxide into equal amounts of deionized water. (This solution was left undisturbed for at lest $24 \mathrm{~h}$ in order to precipitate all sodium carbonate before use.) Sodium hydroxide $(250 \mathrm{mM})$ mobile phase was made by diluting $13.1 \mathrm{ml}$ of $50 \%$ sodium hydroxide with 11 of deionized water. The sodium acetate mobile phase $(1.0 \mathrm{M})$ was prepared by dissolving $82.0 \mathrm{~g}$ of anhydrous sodium acetate in 11 deionized water. All of the eluents were maintained under nitrogen (3-4 p.s.i.; 1 p.s.i. $=6894.76 \mathrm{~Pa}$ ) to prevent contamination by atmospheric carbon dioxide. All water used in our work was purified with a laboratory water purification system (Barnstead, IA, USA) and was $18 \mathrm{M} \Omega$ $\mathrm{cm}$ in specific resistance or better.

\subsection{Chromatography system}

The chromatography system used for this work was a Dionex BioLC system. The system consisted 
of a GS50 gradient pump (microbore, polyether ether ketone (PEEK)) with on-line degas, an AS50 autosampler and thermal compartment with $25-\mu 1$ injection loop, and an ED50 electrochemical detector equipped with a thin-layer type amperometric cell. The working electrode was made from a 1-mmdiameter gold rod that was force-fitted into a Kel-F plastic block. The counter electrode was the titanium cell body across the $25-\mu \mathrm{m}$ thin-layer channel from the working electrode. A glass and $\mathrm{Ag} / \mathrm{AgCl}$ combination reference electrode (Dionex) was downstream from the thin-layer channel. The IPAD waveform is shown in Table 1. The column was installed in the thermal compartment at a controlled temperature of $30{ }^{\circ} \mathrm{C}$. Chromatography of amino acids and sugars was performed on an AminoPac PA10 analytical column $(250 \times 2 \mathrm{~mm}$, Dionex) at a flow-rate of 0.25 $\mathrm{ml} / \mathrm{min}$. PeakNet 6.30 (Chromeleon) chromatography software (Dionex) was used for system control and data analysis.

\subsection{Preparation of sample solutions}

Two kinds of green tea, various grades of Longjing and Biluochun, were purchased from a local market. An aliquot of $0.5 \mathrm{~g}$ of ground tea leaves was inserted into a flask, $100 \mathrm{ml}$ of hot water $\left(80^{\circ} \mathrm{C}\right)$ were added, then infused for $30 \mathrm{~min}$. The resulting extract solutions were filtered through a $0.45 \mu \mathrm{m}$ membrane filter, and diluted 25 -fold with the diluent

Table 1

Detection waveform for amino acids and sugars

\begin{tabular}{lcl}
\hline Time (s) & $\begin{array}{l}\text { Potential }^{\mathrm{a}}(\mathrm{V}) \\
\text { vs. } \mathrm{pH}\end{array}$ & $\begin{array}{l}\text { Integration } \\
\text { (begin/end) }\end{array}$ \\
\hline 0.00 & 0.13 & \\
0.04 & 0.13 & Begin \\
0.05 & 0.33 & \\
0.21 & 0.33 & \\
0.22 & 0.60 & End \\
0.46 & 0.60 & \\
0.47 & 0.33 & \\
0.56 & 0.33 & \\
0.57 & -1.67 & \\
0.58 & -1.67 & \\
0.59 & 0.93 & \\
0.60 & 0.13 & \\
\hline
\end{tabular}

${ }^{\mathrm{a}}$ Potentials applied to Au working electrode and referenced vs. glass $/ \mathrm{Ag} / \mathrm{AgCl}$ combination electrode. containing $20 \mathrm{mg} / 1$ sodium azide before injection into the system.

\section{Results and discussion}

Previous reports describe separations of amino acids and sugars by AEC-IPAD [14-16]. These methods employed a ternary gradient eluent consisting of water, $0.25 \mathrm{M}$ sodium hydroxide, and $1.0 \mathrm{M}$ sodium acetate as mobile phase. The gradient conditions can be commonly subdivided into three distinct parts: firstly, weakly retained amino acids, monosaccharides and disaccharides are eluted by the hydroxide eluent only. Secondly, a sodium acetate gradient causes the more strongly retained amino acids (such as acidic amino acids and aromatic amino acids) to elute. Thirdly, the initial hydroxide concentration is used to re-equilibrate the column for the next run. Using such gradient conditions (see Table 2, gradient A), we were able to separate amino

Table 2

Gradient conditions for separation of amino acids and sugars

\begin{tabular}{|c|c|c|c|c|}
\hline Gradient & $\begin{array}{l}\text { Time } \\
(\mathrm{min})\end{array}$ & $\begin{array}{l}\mathrm{NaOH} \\
(\mathrm{m} M)\end{array}$ & $\begin{array}{l}\mathrm{NaAc} \\
(\mathrm{m} M)\end{array}$ & Curve $^{a}$ \\
\hline \multirow[t]{10}{*}{ A (Ref. [16]) } & 0.00 & 40 & & \\
\hline & 2.00 & 40 & & \\
\hline & 12.00 & 80 & & 8 \\
\hline & 16.00 & 80 & & \\
\hline & 24.00 & 60 & 400 & 8 \\
\hline & 35.00 & 60 & 400 & \\
\hline & 35.10 & 200 & & 5 \\
\hline & 37.10 & 200 & & \\
\hline & 37.20 & 40 & & 5 \\
\hline & 60.00 & 40 & & \\
\hline \multirow[t]{11}{*}{ B (optimized) } & 0.00 & 5 & & \\
\hline & 8.00 & 5 & & 5 \\
\hline & 16.00 & 60 & & 8 \\
\hline & 18.00 & 80 & & \\
\hline & 22.00 & 60 & & 8 \\
\hline & 30.00 & 60 & 400 & 8 \\
\hline & 40.00 & 60 & 400 & \\
\hline & 40.10 & 200 & & \\
\hline & 42.10 & 200 & & \\
\hline & 42.20 & 5 & & 5 \\
\hline & 67.00 & 5 & & \\
\hline
\end{tabular}

\footnotetext{
${ }^{a}$ Shapes of gradient curves are defined in the GS50 Pump Manual, 37-38 (Dionex document no. 031612, revision 2). Curve 5 is linear and curve 8 is one of the four available concave curves (6-9) with $20 \%$ of change at about $60 \%$ of a time segment and $70 \%$ change at $90 \%$ of the same programmed time segment.
} 
acids, glucose, fructose, and sucrose. However, the peaks of both groups of compounds were eluting close to each other, so any separation of amino acids would have been affected by large amounts of sugars in actual samples. Moreover, theanine, the most important amino acid in green tea, co-eluted with glutamine (see Fig. 1).

\subsection{Effect of sodium hydroxide on retention behavior of amino acids and sugars}

In the initial experiment of this report, we studied the effect of $\mathrm{NaOH}$ on retention behavior of two amino acids (theanine and glutamine) and saccharides (glucose, fructose, and sucrose). A plot of resulting retention times is shown in Fig. 2. As can be seen from the plot, it is useful to decrease the sodium hydroxide concentration to improve the separation between theanine and glutamine. Moreover, the order of elution of these compounds is changed with the variation of $\mathrm{NaOH}$ concentration. At higher concentrations of $\mathrm{NaOH}$ (over $20 \mathrm{mM}$ ), theanine co-elutes with glutamine eluting before sugars, while at concentrations of $\mathrm{NaOH}$ lower than about $8 \mathrm{mM}$, theanine is separated from glutamine and elutes after the three sugars. This change of elution sequence results mainly from different acidic dissociation constants. Because the $\mathrm{p} K_{\mathrm{a}}$ values of

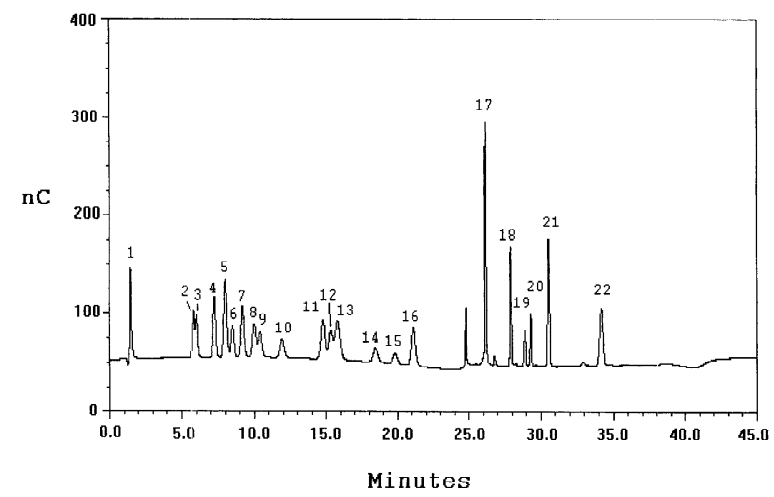

Fig. 1. Separation of standard amino acids and sugars (125 pmol each column) under gradient A. Peaks: 1, arginine; 2, theanine; 3, glutamine; 4, asparagine; 5, glucose; 6 , alanine; 7, threonine; 8 , fructose; 9 , glycine; 10 , valine; 11 , serine; 12 , proline; 13 , sucrose; 14, isoleucine; 15 , leucine; 16, methionine; 17, histidine; 18 , phenylalanine; 19, glutamic acid; 20, aspartic acid; 21, cystine; 22, tyrosine.

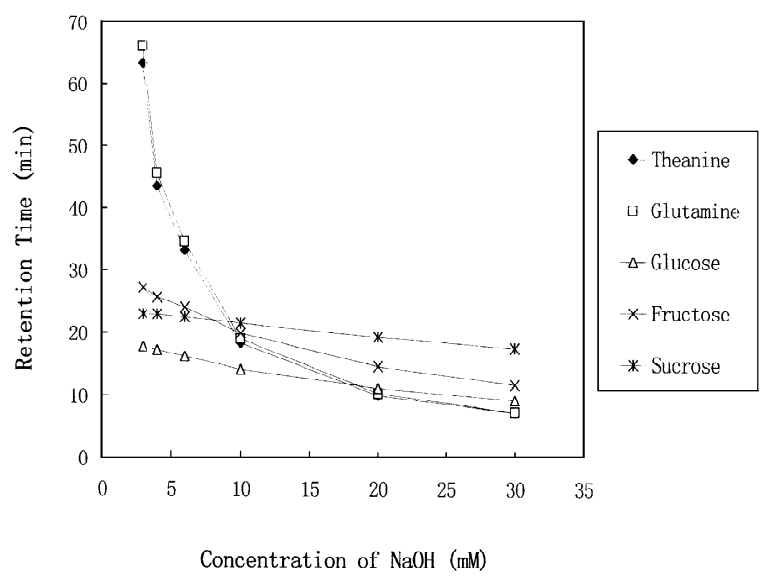

Fig. 2. Effect of $\mathrm{NaOH}$ on the retention times of analytes $(5 \mu M)$ under isocratic conditions.

sugars are higher than 12 , the ionization of sugars is suppressed at lower concentrations of $\mathrm{NaOH}$. This effect compensates for the effect of lower concentrations of $\mathrm{NaOH}$ on the retention times of sugars. On the other hand, the ionization of amino acids with relatively low values of $\mathrm{p} K_{\mathrm{a}}$ (except arginine) is not affected at lower concentrations of $\mathrm{NaOH}$. So with a lower concentration of hydroxide, the retention times of amino acids are increased more than those of sugars. In addition, the position of sucrose also changes relative to other sugars. That may be explained from the fact that disaccharides contain more hydroxyl groups than monosaccharides, resulting in an increased interaction with the resins. As a result, the lower initial concentration of $\mathrm{NaOH}$ is helpful to improve resolution between theanine and glutamine and also makes sugars elute apart from neutral amino acids.

\subsection{Optimization of gradient condition}

\subsubsection{Selecting the initial concentration of sodium hydroxide}

In view of the result of Section 3.1, the initial concentration of $\mathrm{NaOH}$ should be lower than $8 \mathrm{~m} M$ to separate theanine from glutamine. The effect of different initial concentrations of $\mathrm{NaOH}$ in gradient B of Table 2 was tested next. The results are shown in Fig. 3. When the initial concentration of $\mathrm{NaOH}$ was $2.5 \mathrm{~m} M$, three sugars could be eluted and 


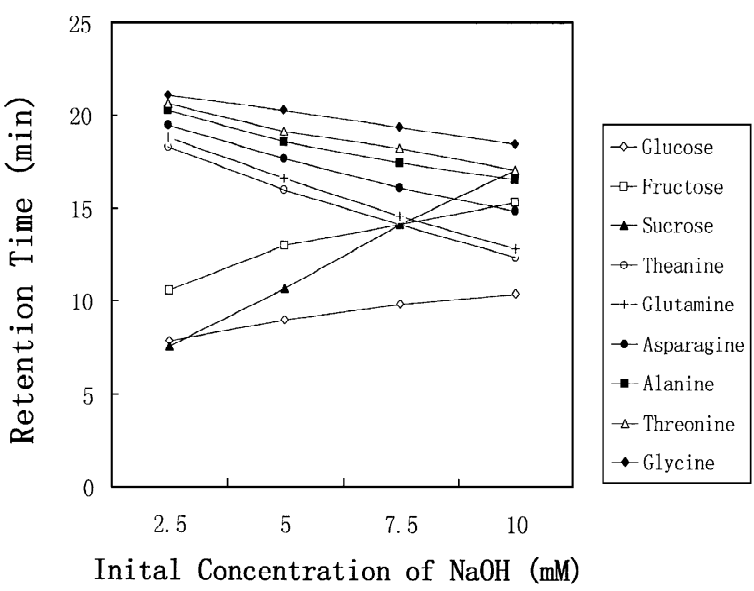

Fig. 3. Effect of initial concentration of $\mathrm{NaOH}$ on the retention time of analytes $(5 \mu M)$ under gradient $\mathrm{B}$ conditions.

separated before theanine, but neutral amino acids eluted close to each other. When the initial concentration of $\mathrm{NaOH}$ was higher than $7.5 \mathrm{mM}$, the peaks of fructose and sucrose were eluting close to or co-eluting with the peaks of neutral amino acids. When the initial concentration of $\mathrm{NaOH}$ was chosen at $5 \mathrm{mM}$, the chromatographic separation of amino acids and sugars was optimal. In addition, 8 min was determined to be a suitable interval of time for maintaining the low initial concentration of $\mathrm{NaOH}$ during the gradient in order to make all sugars elute earlier than amino acids. If that time interval was shorter, the peak of theanine was close to the peak of fructose. On the other hand, when that time interval was longer than $8 \mathrm{~min}$, the peaks of amino acids from theanine to valine were eluting in a very short time interval and their separation was, in our judgement, not sufficient for uneven levels of analytes expected in actual samples.

\subsubsection{Selecting re-equilibration time}

In this section, we discuss the differences in the effect of re-equilibration intervals of gradient B (time interval starting at $42.2 \mathrm{~min}$ in Table $2,5 \mathrm{mM}$ $\mathrm{NaOH})$ on the retention times of sugars and amino acids. As expected [14-16] under the chosen experimental conditions, with re-equilibration intervals exceeding ca. $20 \mathrm{~min}$, retention times of all amino acids become relatively stable. They change only very little if the length of the re-equilibration interval is varied between 20 and $50 \mathrm{~min}$.

In contrast, the retention times of sugars change significantly under the same conditions. With reequilibration times longer than $40 \mathrm{~min}$, the sucrose peak will co-elute closely with peaks of amino acids, while with re-equilibration times shorter than $25 \mathrm{~min}$ the peak of sucrose tends to co-elute with the peak of glucose. Only with a re-equilibration time of 25-30 min is the separation of sugars and amino acids fully optimized (Fig. 4).

By adding, for example, $0.01 M$ sodium acetate into the $5 \mathrm{~m} M$ sodium hydroxide during the time segment between 0 and $8 \mathrm{~min}$, it is possible to obtain similar grouping of peaks as with shorter equilibration intervals (glucose, sucrose co-elution) even after long re-equilibration intervals. We take this observation as evidence that traces of acetate influence the retention of sugars to a greater degree than that of amino acids and the residual acetate concentration can be used to increase the separation of carbohydrates from amino acids. As described in the following section, such residual presence of acetate can be achieved with acceptable reproducibility from run to run. With the use of the optimized gradient $\mathrm{B}$, the chromatogram of amino acids and sugars is shown in Fig. 5.

\subsection{Method validation}

We verified the reproducibility of all retention

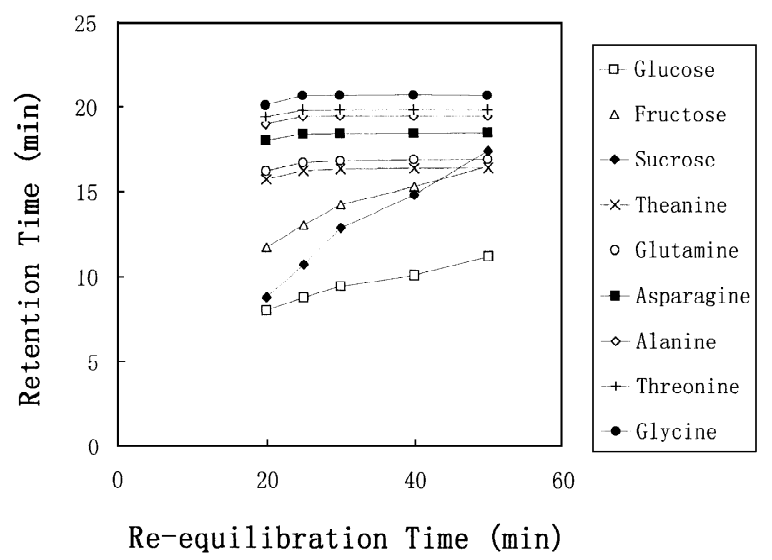

Fig. 4. Effect of the re-equilibration time on the retention time of analytes $(5 \mu M)$ under gradient $\mathrm{B}$ conditions. 


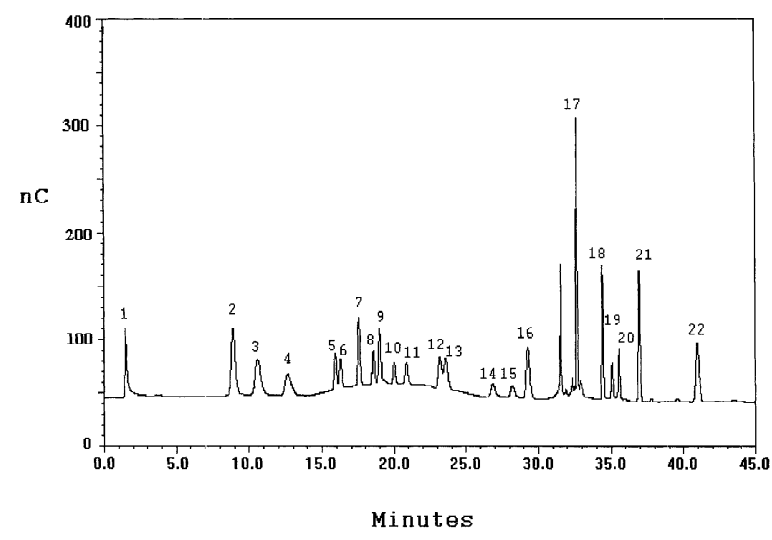

Fig. 5. Separation of standard amino acids and sugars (125 pmol each column) under gradient B conditions. Peaks: 1, arginine; 2, glucose; 3, sucrose; 4, fructose; 5, theanine; 6, glutamine; 7, asparagine; 8 , alanine; 9, threonine; 10, glycine; 11, valine; 12, serine; 13 , proline; 14 , isoleucine; 15 , leucine; 16 , methionine; 17 , histidine; 18, phenylalanine; 19, glutamic acid; 20, aspartic acid; 21, cystine; 22, tyrosine. times (nine injections, $\mathrm{RSD}<1.7 \%$ ) using the reequilibration interval of $25 \mathrm{~min}$. Reproducibility of all peak areas was in the range of 0.8 to $4.6 \%$ under the same conditions. The summary of validation measurements is presented in Table 3 . The plots of peak areas vs. concentration were linear over 2-3 orders of magnitude above the detection limit for each of the analytes. The correlation coefficients for most amino acids and for all sugars were better than 0.99 over three orders of magnitude. The linear ranges for the basic amino acids and theanine were narrow resulting in correlation coefficients $<0.99$ over two orders of magnitude. The difference in calibration plots between basic amino acids and all other compounds analyzed by AEC-IPAD are explainable from the description of the detection mechanism provided in the literature [14].

Limits of detection were evaluated as three times the noise. Similarly as in previous reports using different gradient conditions [14], in our study,

Table 3

Linearity, detection limit and precision of the method

\begin{tabular}{|c|c|c|c|c|c|}
\hline \multirow[t]{2}{*}{ Compounds } & \multicolumn{2}{|l|}{ Linearity } & \multirow{2}{*}{$\begin{array}{l}\text { Detection limit }{ }^{\mathrm{a}} \\
(\mathrm{pmol})\end{array}$} & \multicolumn{2}{|c|}{ Precision (RSD, $n=9)(\%)$} \\
\hline & Range & $r$ & & Peak area & Retention time \\
\hline Arginine & $0.05-10$ & 0.9972 & 0.50 & 1.2 & 0.5 \\
\hline Glucose & $0.05-50$ & 0.9991 & 0.25 & 1.4 & 1.0 \\
\hline Sucrose & $0.05-50$ & 0.9985 & 0.40 & 1.6 & 1.7 \\
\hline Fructose & $0.05-50$ & 0.9962 & 0.80 & 1.1 & 1.2 \\
\hline Theanine & $0.1-10$ & 0.9927 & 1.20 & 1.5 & 1.0 \\
\hline Glutamine & $0.1-25$ & 0.9918 & 1.16 & 1.4 & 1.0 \\
\hline Asparagine & $0.05-25$ & 0.9924 & 0.46 & 1.5 & 0.6 \\
\hline Alanine & $0.05-20$ & 0.9952 & 1.22 & 2.5 & 0.4 \\
\hline Threonine & $0.1-25$ & 0.9969 & 0.47 & 0.8 & 0.4 \\
\hline Glycine & $0.05-50$ & 0.9999 & 0.60 & 1.9 & 0.3 \\
\hline Valine & $0.1-50$ & 0.9960 & 2.50 & 2.3 & 0.2 \\
\hline Serine & $0.1-50$ & 0.9996 & 1.52 & 0.8 & 0.1 \\
\hline Proline & $0.1-50$ & 0.9991 & 1.50 & 0.8 & 0.1 \\
\hline Isoleucine & $0.1-50$ & 0.9997 & 4.90 & 0.9 & 0.1 \\
\hline Leucine & $0.1-50$ & 0.9999 & 3.58 & 2.2 & 0.2 \\
\hline Methionine & $0.1-50$ & 0.9958 & 2.23 & 1.0 & 0.2 \\
\hline Histidine & $0.05-10$ & 0.9965 & 0.37 & 1.7 & 0.05 \\
\hline Phenylalanine & $0.05-50$ & 0.9938 & 0.58 & 1.6 & 0.04 \\
\hline Glutamic acid & $0.5-50$ & 0.9994 & 0.44 & 4.6 & 0.2 \\
\hline Aspartic acid & $0.5-50$ & 0.9993 & 0.48 & 4.5 & 0.2 \\
\hline Cystine & $0.05-50$ & 0.9981 & 0.12 & 0.6 & 0.1 \\
\hline Tyrosine & $0.05-50$ & 0.9992 & 0.41 & 1.3 & 0.1 \\
\hline
\end{tabular}

\footnotetext{
${ }^{\mathrm{a}}$ Injection volume $25 \mu \mathrm{l}$.
} 


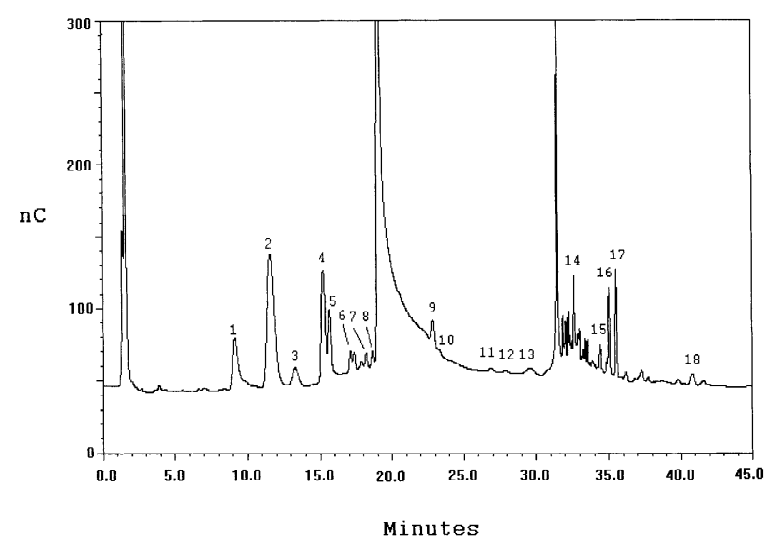

Fig. 6. Chromatographic separation of amino acids and sugars in green tea infusion (the high grade of Longjing). Peaks: 1, glucose; 2 , sucrose; 3 , fructose; 4 , theanine; 5 , glutamine; 6 , asparagine; 7 , alanine; 8, threonine; 9, serine; 10, proline; 11, isoleucine; 12, leucine; 13, methionine; 14, histidine; 15, phenylalanine; 16, glutamic acid; 17, aspartic acid; 18 , tyrosine.

amino acids containing nonpolar side chains (leucine, isoleucine, methionine, and valine) also exhibited slightly higher limits of detection than the remaining analytes.

\subsection{Sample analysis}

The usefulness of the new gradient method (Table 2 , gradient B) was tested on various grades of green tea. A chromatogram of green tea (high grade Longjing) is presented in Fig. 6. It can be seen that in addition to amino acids, sugars are also present in the analyzed sample of green tea. With the exception of theanine, glutamine, glutamic acid, and aspartic acid, other amino acids are present only at trace levels in the chromatogram of Fig. 6.

We performed a recovery test for each of the analytes identified in three different grades of two major types of green tea. This was done by spiking with standards at 50 to $100 \%$ of the determined level and repeating each spiking experiment three times. The average recoveries ranged from 92.12 to $103.8 \%$. The results of the recovery study and sample analysis are presented in Table 4.

\section{Conclusion}

A simple, rapid and sensitive method was de-

Table 4

The results of the recovery and analysis for green tea

\begin{tabular}{|c|c|c|c|c|c|c|c|}
\hline \multirow[t]{3}{*}{ Compounds } & \multirow{3}{*}{$\begin{array}{l}\text { Recovery } \\
(\%) n=3 \\
\text { Mean } \pm \text { SD }\end{array}$} & \multicolumn{6}{|c|}{ Content $(\mathrm{mg} / \mathrm{g})$ in dried fresh tea leaf } \\
\hline & & \multicolumn{3}{|c|}{ Longjing } & \multicolumn{3}{|c|}{ Biluochun } \\
\hline & & High & Medium & Low & High & Medium & Low \\
\hline Glucose & $99.56 \pm 1.7$ & 0.98 & 0.90 & 0.59 & 1.31 & 1.06 & 0.73 \\
\hline Sucrose & $98.24 \pm 3.1$ & 9.94 & 8.29 & 8.20 & 7.81 & 7.20 & 6.16 \\
\hline Fructose & $99.23 \pm 2.9$ & 0.86 & 0.72 & 0.47 & 0.79 & 0.40 & 0.38 \\
\hline Theanine & $98.31 \pm 2.4$ & 10.73 & 9.83 & 9.16 & 9.91 & 9.08 & 8.51 \\
\hline Glutamine & $99.22 \pm 2.7$ & 2.24 & 2.21 & 1.99 & 3.11 & 1.67 & 1.66 \\
\hline Asparagine & $103.7 \pm 1.5$ & 0.33 & 0.27 & 0.84 & 0.25 & 0.38 & 0.50 \\
\hline Alanine & $97.90 \pm 1.9$ & 0.33 & 0.33 & 0.30 & 0.31 & 0.29 & 0.25 \\
\hline Threonine & $97.23 \pm 3.1$ & 0.27 & 0.34 & 0.45 & 0.23 & 0.29 & 0.28 \\
\hline Serine & $103.8 \pm 3.2$ & 0.69 & 0.63 & 0.76 & 0.55 & 0.51 & 0.47 \\
\hline Proline & $99.10 \pm 2.9$ & 0.20 & 0.18 & 0.28 & 0.11 & 0.16 & 0.17 \\
\hline Isoleucine & $96.34 \pm 2.2$ & 0.10 & $>$ LOD & $>$ LOD & $>$ LOD & $>$ LOD & 0.10 \\
\hline Leucine & $97.61 \pm 2.5$ & 0.32 & 0.20 & 0.36 & 0.21 & 0.23 & 0.29 \\
\hline Methionine & $98.74 \pm 3.0$ & 0.10 & $>$ LOD & $>$ LOD & $>$ LOD & $>$ LOD & $>\mathrm{LOD}$ \\
\hline Histidine & $92.12 \pm 2.3$ & 0.30 & 0.30 & 0.33 & 0.35 & 0.19 & 0.17 \\
\hline Phenylalanine & $98.67 \pm 3.1$ & 0.26 & 0.25 & 0.56 & 0.13 & 0.23 & 0.30 \\
\hline Glutamic acid & $102.5 \pm 3.6$ & 3.05 & 3.06 & 2.76 & 3.13 & 2.67 & 2.34 \\
\hline Aspartic acid & $103.4 \pm 3.9$ & 2.21 & 2.44 & 2.12 & 2.67 & 2.35 & 2.00 \\
\hline Tyrosine & $99.06 \pm 4.1$ & 0.36 & 0.34 & 0.50 & 0.30 & 0.35 & 0.39 \\
\hline
\end{tabular}


veloped for the analysis of amino acids and sugars in green tea. The new method does not require any sample derivatization. Amino acids and sugars can be separated without any interference with each other. Especially, theanine and glutamine are well separated from each other and can both be detected with high sensitivity. This method thus fulfils all requirements as a robust and simple technique for evaluation of green tea quality based on amino acid and carbohydrate profiling.

\section{References}

[1] S. Kato, T. Suzuki, J. Food Sci. Technol. 18 (1971) 388.

[2] M. Nakagawa, I. Amano, J. Food Technol. 21 (1974) 57.

[3] H. Horie, K. Kohata, J. Chromatogr. A 881 (2000) 425.

[4] T. Mukai, H. Horie, T. Goto, Tea Res. J. 76 (1992) 45.
[5] D.C. Chu, K. Kobayashi, L.R. Juneja, T. Yamamoto, in: T. Yamamoto, L.R. Juneja, D.C. Chu, M. Kim (Eds.), Chemistry and Application of Green Tea, CRC Press, Boca Raton, FL, 1997, p. 8.

[6] K.H. Ekborg-Ott, A. Taylor, D.W. Armostrong, J. Agric. Food Chem. 45 (1997) 353.

[7] H. Yokogoshi, Y. Kato, Y.M. Sagesaka et al., Biosci. Biotechnol. Biochem. 59 (1995) 615.

[8] T. Sugiyama, Y. Sadzuka, Clin. Cancer Res. 5 (1999) 413.

[9] K. Ohtsuki, M. Kawabata, H. Kokura, K. Taguchi, Agric. Biol. Chem. 51 (1987) 2479.

[10] T. Goto, H. Horie, T. Mukai, Tea Res. J. 71 (1993) 29.

[11] K. Ohta, A. Yoshida, K. Harada, J. Agric. Chem. Soc. Jpn. 69 (1995) 1331

[12] H. Horie, K. Kohata, J. Chromatogr. A 802 (1998) 219.

[13] J.P. Aucamp, Y. Hara, Z. Apostolides, J. Chromatogr. A 876 (2000) 235.

[14] A.P. Clarke, P. Jandik, R.D. Rocklin, Anal. Chem. 71 (1999) 2774.

[15] P. Jandik, J. Cheng, D. Jensen, J. Chromatogr. B 758 (2001) 189.

[16] P. Jandik, A.P. Clark, N. Avdalovic, J. Chromatogr. B 732 (1999) 193. 